\title{
Heartbeat: What is the best emergency treatment for decompensated severe aortic stenosis?
}

The cornerstone of treatment for patients with severe aortic stenosis (AS) is prompt valve replacement as soon as even mild symptoms are present. Even so, many patients are referred much later in the disease course, either because the primary care provider did not make the correct diagnosis or erroneously assumed the patient was 'too old' or 'too sick' to undergo valve replacement. In this situation, transfer to a heart valve centre often occurs only when the patient becomes haemodynamically unstable. Optimal emergency management of decompensated severe AS is controversial-attempts at medical stabilisation typically are futile, surgical risk is prohibitive and the decision to perform balloon aortic valvuloplasty (BAV) or transcatheter aortic valve implantation (TAVI) is not straightforward.

In this issue of Heart, Bongiovanni and colleagues ${ }^{1}$ report the outcomes of 141 patients with decompensated severe AS treated with TAVI $(n=23)$ or BAV $(n=118)$. Procedural mortality was $8.7 \%$ for TAVI compared with $20.3 \%$ for BAV with 30-day cardiovascular mortality rates of $23.8 \%$ for TAVI and $33.0 \%$ for BAV (figure 1). These differences are not statistically significant and were not affected by potential confounders. The subgroup of patients who underwent TAVI after an initial BAV $(n=32)$ still had high procedural (9.4\%) and 30-day (15.6\%) mortality rates. Compared with BAV, TAVI was associated with more major vascular complications and strokes.

In the accompanying editorial, Adamson and Cruden ${ }^{2}$ discuss the challenges of management in patients with decompensated severe AS. They emphasise that: "Central to this decision making is the multidisciplinary Heart Team through a careful and thorough assessment to evaluate whether aortic stenosis is the principal cause of decompensation, comorbid status, technical factors and prognosis." However, they conclude: "where technically feasible, in carefully selected patients with minimal comorbidity and an

Correspondence to Professor Catherine M Otto, Division of Cardiology, University of Washington, Seattle,WA 98195, USA; cmotto@uw.edu

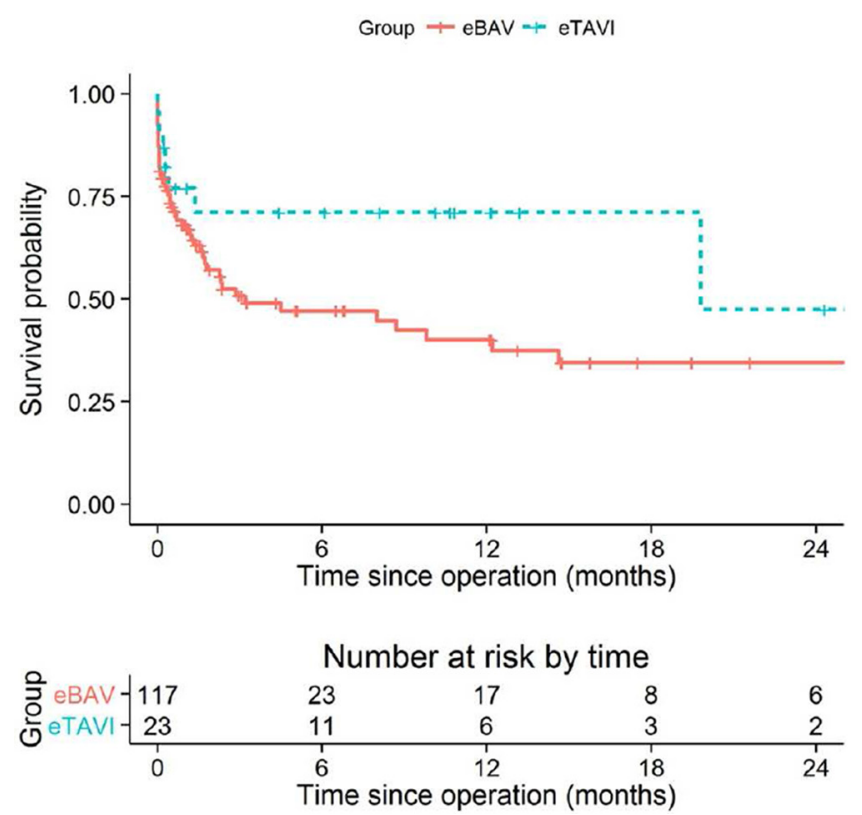

Figure 1 Kaplan-Meier survival curves for eBAV and eTAVI patients (restricted to the first 2 years of follow-up). The eBAV patients with subsequent elective TAVI were censored at the time of TAVI intervention. Corresponding Cox models were fitted. The estimated HR comparing eTAVI patients with eBAV is $0.59(0.28 ; 1.25), P=0.169$. The estimated $H R$ is comparable following adjustment for age, logistic Euroscore, atrial fibrillation, coronary artery disease and a $\mathrm{PCl}$ in the 30 days preceding the emergency procedure: $0.45(0.19,1.08), P=0.074$. eBAV, emergency balloon valvuloplasty; eTAVI, emergency transcatheter aortic valve implantation; TAVI, transcatheter aortic valve implantation.

otherwise favourable prognosis, a strategy of up-front emergency TAVI may be considered. For the majority of patients, however, BAV affords the opportunity to palliate symptoms, assess treatment response and clarify comorbid status and

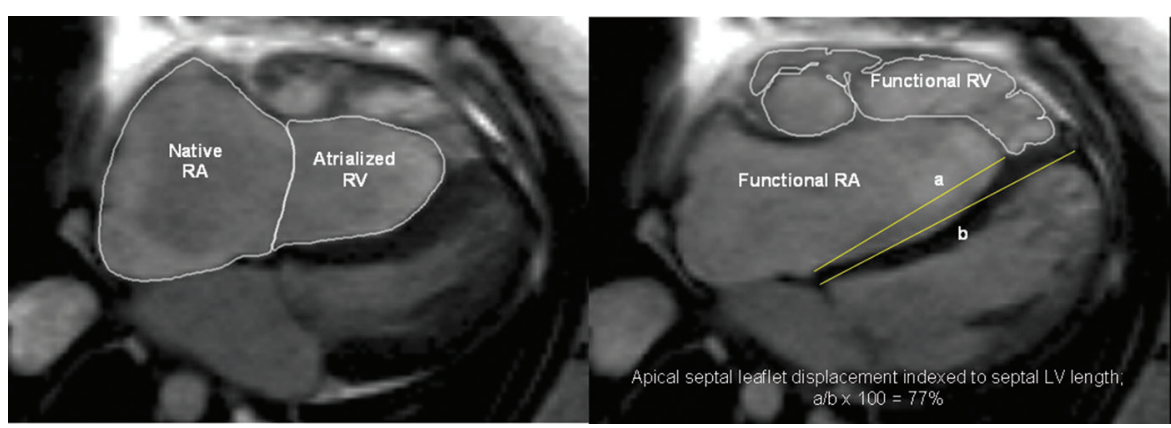

Figure 2 Measurements of native right atrial (RA) and atrialised right ventricular (RV) volumes, functional RV volumes and apical septal leaflet displacement/left ventricular (LV) septal length $(A / B * 100 \%)$. Steady-state free precession sequence and axial stack were used. prognosis." No doubt, this controversy is not yet over.

Among the many factors that predict longevity, one of the simplest to measure is resting heart rate (RHR), which is associated with a 


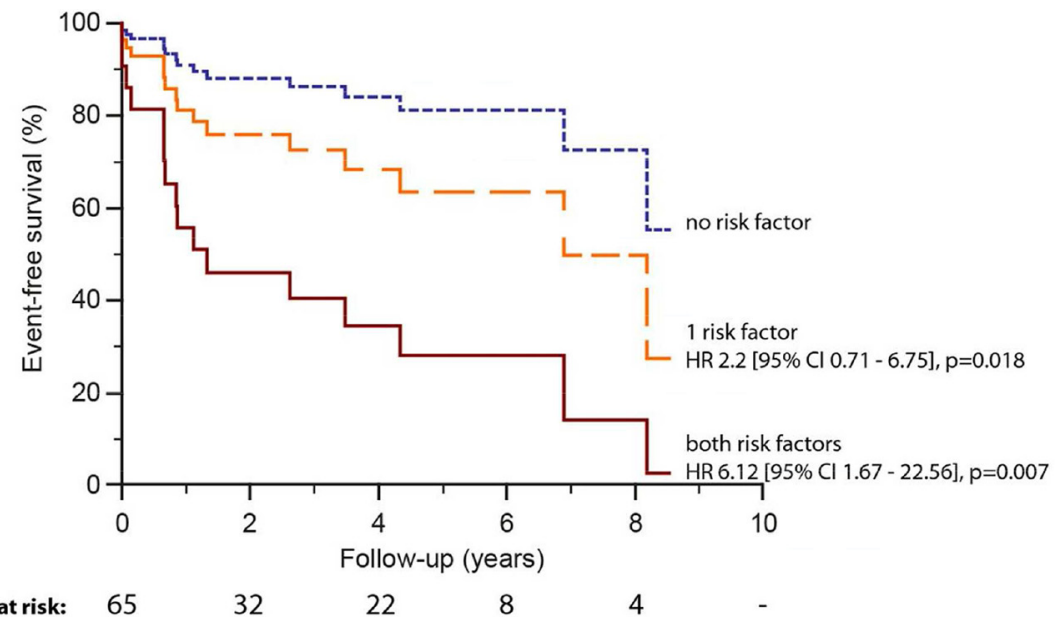

Figure 3 Survival curves for first-onset atrial tachycardia $(n=65)$ stratified by univariable predictors with upper quartile functional right ventricular:left ventricular (LV) ratio (>2.4) and apical septal leaflet displacement/LV septal length (>67\%) cut-offs accordingly.

$30 \%-50 \%$ excess mortality for every $20 \mathrm{bpm}$ increase in RHR. Although RHR depends partly on extrinsic factors, such as physical conditioning, there also is a heritable component. In a study of 4282 twins without cardiovascular disease, overall hereditability estimates were 0.23 (95\% CI 0.15 to 0.30 ) overall; 0.27 (95\% CI 0.15 to 0.38 ) for males and 0.17 (95\% CI 0.06 to 0.28 ) for females. ${ }^{3}$

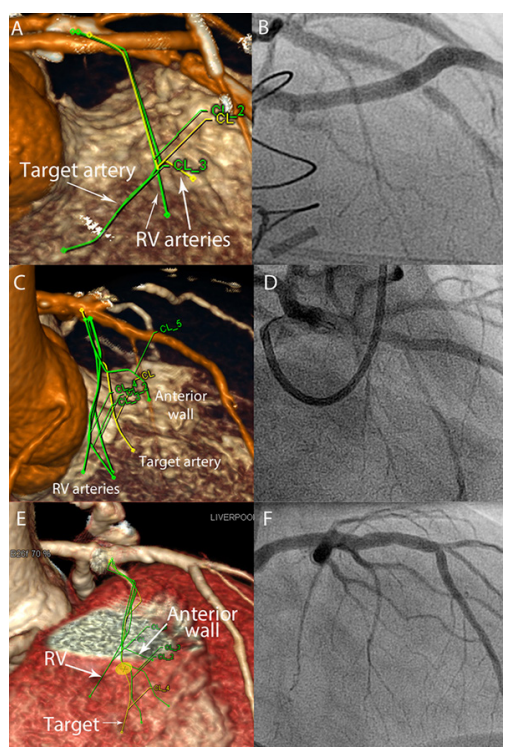

Figure 4 (A) CT angiogram. The traced septal vessels from two-dimensional images were projected on to the coronary angiogram 'map'. This CT angiogram is rotated to minimise foreshortening and remove overlap (in this example to RAO cranial). The equivalent invasive angiogram projection is shown in (B). The target artery is identified and only this subbranch is occluded for alcohol delivery. Further examples are shown in $(C, D)$ and $(E, F)$. RAO, right anterior oblique; $\mathrm{RV}$, right ventricular. rial by Munroe and Tinker. ${ }^{4}$ The current study adds to this data by demonstrating mortality, even after adjustment for cular mortality hazard ratio of 2.19 (1.30 to 3.67$)$ for a RHR of $>90$ compared with 61-70 bpm.

The association between RHR and mortality and previous smaller studies on transoesophageal echocardiography. that the genetic heritability of RHR is maintained, despite effects of environmental factors, such as patients age. They conclude: "A maintained heritability of RHR supports the continued search for genetic factors underlying RHR to provide further insights into understanding the key physiological mechanisms explaining variation in RHR.” As we put this data in the context of other studies suggesting that a lower RHR in men might be associated with an increased risk of atrial fibrillation, we clearly have more to learn about RHR as a predictor of cardiovascular outcomes.

Advanced cardiac imaging has the potential to improve care of patients with complex cardiac disease. This is illustrated by a study of cardiac magnetic resonance (CMR) imaging in 79 adults with Ebstein's anomaly of the tricuspid valve. $^{5}$ CMR predictors of mortality (3.4 years follow-up) were right (RV) and left ventricular (LV) ejection fraction, LV stroke volume index and cardiac index (figure 2). Atrial tachycardia (AT) occurred in $22 \%$ and often preceded other adverse events. CMR predictors of AT were RV ejection fraction, the ratio of $\mathrm{RV}$ to $\mathrm{LV}$ volumes and the degree of apical displacement of the septal tricuspid valve leaflet (figure 3 ). This study demonstrates the value of CMR imaging to identify patients at risk

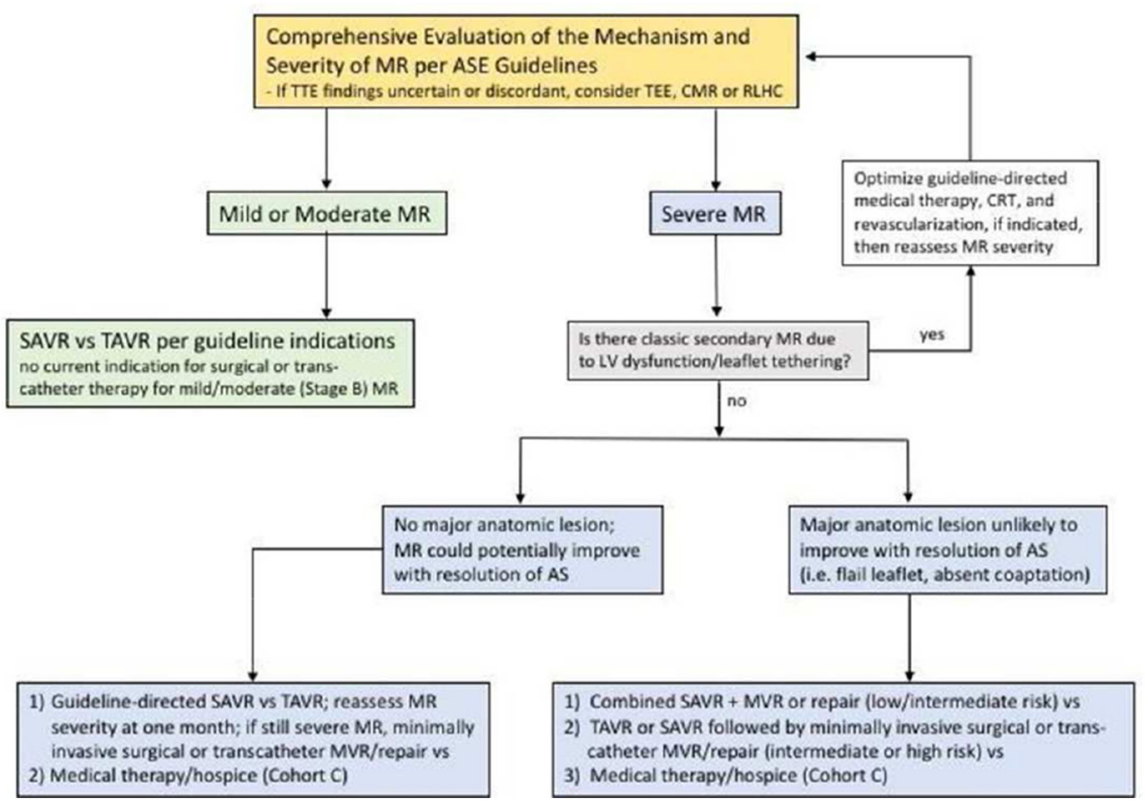

Figure 5 Proposed algorithm for the evaluation and management of patients with severe aortic stenosis (AS) and concomitant mitral regurgitation (MR). ASE, American Society of Echocardiography; CMR, Cine Magnetic Resonance; CRT, cardiac resynchronization therapy; $\mathrm{LV}$, left ventricular; MVR, mitral valve replacement; RHLC, right and left heart catheterisation; SAVR, surgical aortic valve replacement; TAVR, transcatheter aortic valve replacement; TEE, 
adverse cardiac events, potentially justifying earlier intervention for structural disease. The importance of AT as a marker of increased risk also suggests that earlier or more aggressive arrhythmia prevention and management might be beneficial. The authors proposed composite of several CMR factors, in conjunction with other elements of shared decision making, is eminently reasonable.

The Education in Heart article ${ }^{6}$ in this issue, discusses non-surgical septal reduction therapy for relief of subaortic outflow obstruction in adults with hypertrophic cardiomyopathy. This article includes indication, imaging approaches, technical aspects of the procedure itself and outcome data (figure 4).

A review article in this issue by Sannino and Grayburn ${ }^{7}$ on diagnosis and management of mitral regurgitation in adults with
AS is not to be missed. The dilemma is whether to treat both AS and mitral regurgitation at the same time or whether to treat AS first to see if mitral regurgitation improves. In the interventional era, many patients have both valve lesions and the answer is not easy (figure 5).

Competing interests None declared.

Provenance and peer review Commissioned; internally peer reviewed.

(C) Article author(s) (or their employer(s) unless otherwise stated in the text of the article) 2018. All rights reserved. No commercial use is permitted unless otherwise expressly granted.

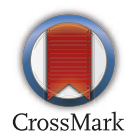

To cite Otto CM. Heart 2018;104:1-3.

Published Online First 1 January 2018
Heart 2018;104:1-3.

doi:10.1136/heartjnl-2017-312661

\section{REFERENCES}

1 Bongiovanni D, Kühl C, Bleiziffer S, et al. Emergency treatment of decompensated aortic stenosis. Heart 2018;104:23-9.

2 Adamson PD, Cruden N. Emergency interventions for the treatment of decompensated aortic stenosis. Heart 2018;104:4-5.

3 Jensen MT, Wod M, Galatius S, et al. Heritability of resting heart rate and association with mortality in middle-aged and elderly twins. Heart 2018;104:30-6.

4 Munroe PB, Tinker A. Heritability of resting heart rate and association with mortality in middle-aged and elderly twins. Heart 2018;104:6-7.

5 Rydman R, Shiina Y, Diller GP, et al. Major adverse events and atrial tachycardia in Ebstein's anomaly predicted by cardiovascular magnetic resonance. Heart 2018; 104:37-44

6 Cooper RM, Stables RH. Non-surgical septal reduction therapy in hypertrophic cardiomyopathy. Heart 2018;104:73-83.

7 Sannino A, Grayburn PA. Mitral regurgitation in patients with severe aortic stenosis: diagnosis and management. Heart 2018;104:16-22. 